\title{
PRESERVAÇÃO DOS ATRIBUTOS FÍSICOS DE FRUTOS DE ATEMOIA cV. GEFNER COM O USO DE 1-MCP E ATMOSFERA MODIFICADA ${ }^{1}$
}

\author{
GLÁUCIA MICHELLE COSME SILVA², MARLON ALTOÉ BIAZATTI², \\ MÍRIAN PEIXOTO SOARES DA SILVA ${ }^{3}$, MARIA HELENA MENEZES CORDEIRO5, \\ GISELE POLETE MIZOBUTSI ${ }^{6}$
}

RESUMO - O interesse comercial pela atemoia vem aumentando cada vez mais no Brasil. O aumento da atividade respiratória, acompanhado por modificações rápidas na composição física dos frutos podem inviabilizar sua distribuição a mercados distantes. Neste contexto, objetivou-se com o presente trabalho avaliar os atributos físicos em frutos de atemoia tratados com 1-metilciclopropeno (1-MCP) e atmosfera modificada associados à refrigeração. $\mathrm{O}$ experimento foi instalado em delineamento experimental inteiramente casualizado, em esquema fatorial 4x5, sendo quatro concentrações de 1-MCP $\left(0 ; 200 ; 400\right.$ e $\left.600 \mathrm{yL} \mathrm{L}^{-1}\right)$ e cinco períodos de avaliação após a colheita, em intervalo de cinco dias, com quatro repetições e quatro frutos por unidade experimental. As atemoias foram colhidas em pomar comercial, no município de Matias Cardoso-MG, no estádio de maturação fisiológica, lavadas, sanitizadas e secas ao ar, depois tratadas com as concentrações de 1-MCP por oito horas, em temperatura ambiente. Logo após, quatro frutos foram dispostos em bandejas de poliestireno expandido. Alguns permaneceram sem membrana, enquanto outros foram embalados com membrana plástica de PEBD $16 \mu \mathrm{m}$. As variáveis avaliadas nos frutos foram: perda de biomassa fresca, firmeza, concentração de $\mathrm{CO}_{2}$, etileno e coloração da casca. O uso da atmosfera modificada e do 1-MCP, associados ou não, foi eficiente no atraso do amadurecimento dos frutos, permitindo a conservação de sua qualidade física. Frutos tratados com 1-MCP apresentaram-se mais firmes, além da preservação da coloração, verificada através da luminosidade, cromaticidade e ângulo Hue ${ }^{\circ}$, que se mostraram superiores quando comparados aos frutos não tratados.

Termos para indexação: Annona squamosa x Annona cherimola, amadurecimento, qualidade física, póscolheita, etileno.

\section{PRESERVATION IN PHYSICAL ATTRIBUTES OF ATEMOYA cv. GEFNER FRUIT WITH THE USE OF 1-MCP AND MODIFIED ATMOSPHERE}

\begin{abstract}
The commercial interest by atemoya is increasing more and more in Brazil. The increased respiratory activity accompanied by rapid changes in the physical composition of the fruits may prevent their distribution to distant markets. In this context, the aim of this study was to evaluate the physical attributes in atemoya fruits treated with 1-methylcyclopropene (1-MCP) and modified atmosphere associated with refrigeration. The experiment was carried out in a randomized design, in a factorial $4 \times 5$, four concentrations of 1-MCP $\left(0,200,400\right.$ and $\left.600 \mathrm{~nL} \mathrm{~L}-^{-1}\right)$, and five evaluation periods after harvest, in the range of five days, with four replications and four fruits per experimental unit. The atemoya were harvested, from a commercial orchard in the municipality of Matias Cardoso, North of Minas Gerais, at the physiological maturity, rinsed, sanitized and air dried, then treated with concentrations of 1-MCP for eight hours at room temperature. Soon after, four fruits were placed in polystyrene trays. Some remained without membrane, while others were packed with plastic film of LDPE $16 \mu \mathrm{m}$. The variables evaluated in the fruits were: fresh biomass loss, firmness, concentration of $\mathrm{CO}_{2}$, ethylene and peel color. The use of modified atmosphere and 1-MCP alone or combined, were effective in delaying fruits ripening allowing the preservation of its physical quality. Fruits treated with 1-MCP were firmer, as well as preservation of staining, verified by luminosity, chroma and $\mathrm{Hue}^{\circ}$ angle, which proved to be superior when compared to untreated fruits.
\end{abstract}

Index terms: Annona squamosa $\mathrm{x}$ Annona cherimola, ripening, physical quality, postharvest, ethylene.

\footnotetext{
'(Trabalho 317-13). Recebido em:

${ }^{2}$ Doutorandos em Produção Vegetal, UENF/CCTA, Campos dos Goytacazes - RJ. E-mails: glaucia-michelle@hotmail.com; marlonbiazatti@hotmail.com

${ }^{3}$ Doutora em Produção Vegetal, UENF/CCTA, Campos dos Goytacazes - RJ. E-mail: mirianpsoares@gmail.com;

${ }^{4}$ Mestranda em Produção Vegetal, UNIMONTES/CCTA, Janaúba - MG. E-mail: helenaagro@yahoo.com.br

${ }^{5}$ Professora Titular de Fisiologia de Pós-colheita, UNIMONTES/CCTA, Janaúba - MG. E-mail: gisele.mizobutsi@unimontes.br
} 


\section{INTRODUÇÃO}

O Brasil possui condições favoráveis para o cultivo de diversas frutíferas, e dentre elas, está a atemoia (Annona squamosa x Annona cherimola), um fruto híbrido pertencente à família Annonaceae, proveniente do cruzamento entre um fruto tropical, a fruta-do-conde (A. squamosa), e um fruto subtropical, a cherimólia (A. cherimola) (FIRMINO et al., 2012).

Apesar de muito apreciada, a comercialização da atemoia está restrita ao mercado interno, principalmente pelo fato de este fruto possuir baixa vida útil pós-colheita, atribuído à sua alta perecibilidade (LIMA et al., 2010; MIZOBUTSI et al., 2012). Esta característica pode ser ainda mais agravada quando estes frutos são submetidos a danos durante a colheita, transporte, manuseio e quando armazenados por longos períodos, representando um entrave à manutenção de sua qualidade. Algumas alterações físicas, como a rápida perda de firmeza, e mudanças na coloração, decorrentes do processo natural de amadurecimento, são fatores observados pelos consumidores (SILVA; MUNIZ, 2011) e que acabam depreciando os frutos.

Para aumentar o período de comercialização destes frutos, algumas tecnologias podem ser adotadas, como as que visam à inibição da ação do etileno, um hormônio natural responsável por regular a maturação e a senescência de frutos, devido à alteração da expressão de genes responsáveis por vários processos fisiológicos, dentre os quais, a respiração climatérica e as mudanças da textura, cor, sabor e aroma (WATKINS, 2006). O 1Meticiclopropeno (1-MCP), inibidor da ação do etileno, tem sido alvo de pesquisas devido aos bons resultados na preservação da qualidade de alguns frutos como: damasco (De MARTINO et al., 2006), atemoia cv. African Pride (LIMA et al., 2010), kiwi (VIEIRA et al., 2010), quivi cv. Hayward (VIEIRA et al., 2012) e pitaia (COCK et al., 2013).

Outra técnica também empregada no intuito de prolongar a vida útil pós-colheita dos frutos é a atmosfera modificada. A utilização de películas plásticas envolvendo os frutos proporciona a manutenção da umidade relativa dentro da embalagem, reduzindo as perdas de massa dos frutos, as taxas respiratórias e a produção de etileno (SILVA et al., 2009; HOJO et al., 2011; MIZOBUTSI et al., 2012).

Diante do exposto, objetivou-se com o presente trabalho avaliar a influência da aplicação pós-colheita de 1-MCP e atmosfera modificada em frutos de atemoia cv. Gefner, associados à refrigeração, sobre seus atributos físicos, visando à manutenção de qualidade e ao prolongamento do tempo de conservação.

\section{MATERIAL E MÉTODOS}

Os frutos de atemoia cv. Gefner foram colhidos em estádio de maturidade fisiológica, em pomar comercial, no município de Matias CardosoMG, localizado à latitude $14^{\circ} 51^{\prime} 17^{\prime}$ 'S, longitude $43^{\circ} 55^{\prime} 19^{\prime}$ 'W, altitude de $472 \mathrm{~m}$, temperatura média anual de $24,2^{\circ} \mathrm{C}$, com precipitação de $870 \mathrm{~mm}^{2} \mathrm{ano}^{-1} \mathrm{e}$ clima do tipo Aw, pela classificação de Köeppen, com chuvas de verão e períodos secos bem definidos no inverno (MOTA FILHO et al., 2012). O delineamento adotado foi o inteiramente casualizado, em esquema fatorial 4x5, sendo quatro concentrações de 1-MCP (0; 200; 400 e $\left.600 \mathrm{gL} \mathrm{L}^{-1}\right)$, e cinco períodos de avaliação após a colheita, em intervalo de 5 dias, com quatro repetições e quatro frutos por unidade experimental.

Após a colheita, os frutos foram selecionados, lavados e sanitizados em solução de hipoclorito de sódio a 1\%, durante cinco minutos. Posteriormente, foram imersos em solução de Sportak (450 CE 450 $\mathrm{g} \mathrm{L}^{-1}$ ) com $15 \mathrm{~mL} \mathrm{~L}^{-1}$ por cinco minutos e colocados para secar ao ar. Em seguida, os frutos foram colocados em caixas plásticas herméticas de $0,2 \mathrm{~m}^{3} \mathrm{e}$ tratados com as respectivas concentrações de 1-MCP por 8 horas, à temperatura ambiente. Uma parte destes frutos foi embalada com polietileno de baixa densidade (PEBD) de $16 \mu \mathrm{m}$, enquanto a outra parte foi acondicionada em bandejas de isopor em número de quatro frutos. Ambos foram armazenados a 15 $\pm 1^{\circ} \mathrm{C}$ e $94 \% \pm 5 \%$ de umidade relativa em câmara fria, onde permaneceram até o final das avaliações.

A perda de biomassa fresca (PBF) foi determinada pela diferença entre a matéria fresca inicial e o valor obtido a cada intervalo de amostragem, expresso em porcentagem. A porcentagem de PBF foi estudada a partir da equação:

$$
\operatorname{PBF}(\%)=\left[\frac{P i-P j}{P i}\right] * 100
$$

Em que: $\mathrm{PBF}=$ perda de biomassa fresca $(\%) ; \mathrm{Pi}=$ peso inicial do fruto $(\mathrm{g})$, e $\mathrm{Pj}=$ peso do fruto no período subsequente a $\mathrm{Pi}(\mathrm{g})$. A firmeza dos frutos (FIR) foi medida nos frutos inteiros, na região mediana, com auxílio do penetrômetro de mesa (FACCHINI, modelo FT 011), utilizando-se de quatro frutos para cada análise. Os resultados foram expressos em Newton $(\mathrm{N})$.

A produção de $\mathrm{CO}_{2}$ e de etileno $\left(\mathrm{C}_{2} \mathrm{H}_{4}\right)$ no interior das embalagens de polietileno de baixa 
densidade foi determinada por cromatografia gasosa, com avaliações feitas a cada 5 dias. Para isso, foram coletadas alíquotas de $1 \mathrm{~mL}$ da atmosfera interna das embalagens. Nos frutos sem embalagem, no mesmo intervalo de avaliação, as atemoias foram acondicionadas em frascos de vidro herméticos com volume de 2,45 L. Sessenta minutos após o fechamento dos frascos, alíquotas de $1 \mathrm{~mL}$ de sua atmosfera foram homogeneizadas e coletadas com uma seringa hermética, sendo estas injetadas em um cromatógrafo a gás (SHIMADZU modelo GC-2014), software GC-Solution. Os cálculos da concentração respiratória foram realizados em bases estequiométricas.

A luminosidade, o ângulo $\mathrm{Hue}^{\circ}$ e cromaticidade foram avaliados por meio de um colorímetro Color Flex 45/0(2200), stdzMode:45/0 com leitura direta de reflectância das coordenadas L (luminosidade), a (variação de cor do verde (-) até o vermelho $(+)$ ), b (variação de cor do azul (-) até o amarelo $(+))$, do sistema Hunterlab Software. Para cada repetição, foi utilizada a média de quatro mensurações por fruto.

Os dados foram submetidos à análise de variância, utilizando-se do programa SANEST (ZONTA et al., 1984), e a regressão polinomial, a $5 \%$ de probabilidade.

\section{RESULTADOS E DISCUSSÃO}

Por meio das equações de regressão apresentadas na Tabela 1, é possível estimar os valores com relação a todas as variáveis testadas, com exceção da cromaticidade, em função do período de armazenamento.

O aumento da dose do 1-MCP, independentemente do uso da embalagem, no decorrer do período de armazenamento, proporcionou menor PBF dos frutos, porém valores ainda menores foram observados quando associados à atmosfera modificada pelo uso de PEBD (Tabela 1).

Hojo et al. (2011) e Mizobutsi et al. (2012), ao testarem diferentes tipos de embalagens plásticas, também observaram menores PBF, em lichia e pinha, respectivamente. Isso pode estar relacionado à redução da transpiração proporcionada pelo filme plástico, responsável pela manutenção da umidade relativa e pelo uso da baixa temperatura que diminui o metabolismo.

O 1-MCP associado à atmosfera modificada pelo uso de PEBD proporcionou baixa PBF, com destaque para a dose de $600 \eta \mathrm{L} \mathrm{L}^{-1}$, sendo possível visualizar a diferença nos valores apresentados: 7,$74 ; 5,23 ; 3,86$ e $2,80 \%$, para as doses de $0 ; 200$;
400 e $600 \eta \mathrm{L} \mathrm{L}^{-1}$, respectivamente, ao final do armazenamento, conferindo a diferença de 176\% entre o controle e a maior dose. Essa mesma dose, também, foi mais eficaz em retardar a PBF para os frutos sem embalagem, apresentando os seguintes valores: 16,$68 ; 11,85 ; 9,57$ e $6,92 \%$, para as doses de $0 ; 200 ; 400$ e $600 \eta \mathrm{L} \mathrm{L}^{-1}$, respectivamente, ao final do armazenamento, conferindo a diferença de $141 \%$ entre o controle e a maior dose utilizada (Tabela 1).

Lima et al. (2010), ao avaliarem a influência da aplicação de 1-MCP $(0 ; 100 ; 200$ e 400 ๆL $\left.\mathrm{L}^{-1}\right)$ sobre a maturação pós-colheita e o tempo de armazenamento ( $0 ; 8$ e 15 dias) sob refrigeração, em atemoia cv. African Pride, relataram que a diferença de $1,3 \%$ de PBF entre os tratamentos não repercutiu em benefício visual nos frutos. No entanto, Valero et al. (2005) relataram reduções na perda de massa de ameixas dependentes das doses aplicadas (250 a $\left.1.000 \eta \mathrm{L} \mathrm{L}^{-1}\right)$.

Com relação à FIR, ao longo do período de armazenamento, houve diferença entre os frutos com e sem embalagem, sendo que estes últimos apresentaram os menores valores. Observou-se que o uso de atmosfera modificada por meio do PEBD contribuiu para atrasar a queda da firmeza que decresceu a partir do $15^{\circ}$ dia, enquanto no tratamento sem embalagem essa queda ocorreu a partir do $5^{\circ}$ dia após a aplicação de 1-MCP. A firmeza dos frutos, no $20^{\circ}$ dia após a colheita, nas doses de $0 ; 200 ; 400$ e 600 $\eta L^{-1}$ de $1-M C P$, foi de 16,$25 ; 13,87 ; 11,59$ e $16,61 \mathrm{~N}$ em frutos sem embalagem e 16,23; 40,02; 54,47 e $69,08 \mathrm{~N}$ nos frutos com embalagem. Isso significa que o 1-MCP possui efeito na retenção da firmeza. A resposta sugere atraso na maturação, com menor degradação dos componentes da parede celular.

Vieira et al. (2012), ao avaliarem frutos de quivi cv. Hayward, também observaram maior conservação da firmeza em frutos. Resultados semelhantes foram encontrados por Dantas (2005), que verificou que pinhas tratadas com 1-MCP, com $400 \mathrm{ppb}$, apresentaram-se mais firmes que as não tratadas. Lima et al. (2010), trabalhando com atemoia cultivar African Pride tratadas com 1-MCP, verificaram diferenças de até $35 \mathrm{~N}$ entre os frutos tratados com 400 nL.L - $^{-1}$ em relação ao controle, aos 15 dias após a colheita. A maior firmeza dos frutos tratados com as maiores doses de 1-MCP está provavelmente associada à redução da atividade das enzimas pectinolíticas, causada pela redução da ação do etileno (JACOMINO et al., 2002).

A taxa respiratória dos frutos sem embalagem foi significativamente maior que a dos frutos com embalagem. Foram observadas que, quanto maior as doses de 1-MCP, menor o pico de produção de 
$\mathrm{CO}_{2} \mathrm{~kg}^{-1} \mathrm{~h}^{-1}$; assim, as doses de $0 ; 200 ; 400$ e 600 $\eta L L^{-1}$ apresentaram valores de 103,88; 92,$57 ; 83,92$ e 76,62 para os frutos sem embalagem e de 95,76 ; 87,$08 ; 84,46$ e $81,99 \mathrm{CO}_{2} \mathrm{~kg}^{-1} \mathrm{~h}^{-1}$ para os frutos com embalagem, respectivamente.

Souza et al. (2008) verificaram redução na atividade respiratória em melão tratado com 1-MCP e armazenado sob refrigeração e atmosfera modificada. Alves et al. (2010) observaram menor atividade respiratória em frutos de kiwis cv. Bruno tratados com 1-MCP a partir do $7^{\circ}$ dia de armazenamento.

A aplicação de 1-MCP reduziu a produção de etileno das atemoias tanto para os frutos sem, quanto os com embalagem. $\mathrm{O}$ tratamento referente à dose de $600 \eta \mathrm{L} \mathrm{L}^{-1}$ de 1-MCP apresentou a menor produção de etileno ao longo do armazenamento, com valor de $52,84 \mathrm{C}_{2} \mathrm{H}_{4} \mathrm{~kg}^{-1} \mathrm{~h}^{-1}$ (no $13^{\circ}$ dia após a colheita) e $42,79 \mathrm{C}_{2} \mathrm{H}_{4} \mathrm{~kg}^{-1} \mathrm{~h}^{-1}$ (no $12^{\circ}$ dia após a colheita), para os frutos sem e com embalagem, respectivamente.

Os valores do ângulo $\mathrm{Hue}^{\circ}$ encontrados na casca da atemoia sem embalagem diminuíram de $101,9^{\circ}$ a $55,32^{\circ} ; 103,5^{\circ}$ a $68,9^{\circ} ; 101,0^{\circ}$ a $78,6^{\circ} ; 101,9^{\circ}$ a $78,42^{\circ}$; do $1^{\circ}$ ao $20^{\circ}$ dia de armazenamento, para as doses de 0;200; 400 e $600 \eta \mathrm{L} \mathrm{L}^{-1}$, respectivamente. Nos frutos com embalagem, os valores do ângulo Hue $^{\circ}$ diminuíram de $102,2^{\circ}$ a $96,6^{\circ} ; 102,4^{\circ}$ a $97,18^{\circ}$; $101,9^{\circ}$ a $98,26^{\circ} ; 97,6^{\circ}$ a $98,8^{\circ}$; do $1^{\circ}$ ao $20^{\circ}$ dia de armazenamento, para as doses de 0;200; $400 \mathrm{e}$ $600 \eta \mathrm{L} \mathrm{L}^{-1}$, respectivamente. Essa mudança indica a evolução da cor da casca da atemoia de verdeescura para verde-clara, a qual variou em todos os tratamentos.

Os frutos com embalagem mudaram de cor mais lentamente, não apresentando diferença significativa entre os tratamentos e os dias de armazenamento, sendo que os frutos sem embalagem atingiram, em menor período, menores valores do ângulo $\mathrm{Hue}^{\circ}$. Isso pode estar relacionado ao fato de a embalagem dificultar trocas gasosas com o meio externo, podendo reduzir a velocidade do metabolismo do fruto. Segundo Brackmann et al. (2006), a presença de $\mathrm{CO}_{2}$ em níveis mais elevados concorre com o etileno pelo seu sítio de ligação no receptor, diminuindo sua ação sobre os mecanismos de síntese e ação das enzimas envolvidas na degradação das clorofilas.

Em relação ao croma $(\mathrm{C})$ ou à cromaticidade, nota-se que, nos frutos sem embalagem, a intensidade de cor diminuiu de 38,67 a $23,49^{\circ}$. Todas as doses de 1-MCP proporcionaram a conservação da intensidade de cor nos frutos com embalagem, quando comparados com os sem embalagem, variando de 32,92 a $16,58^{\circ}$ do $1^{\circ}$ ao $20^{\circ}$ dia de armazenamento (Figura 1).
O valor da coordenada $\mathrm{L}$ nos frutos sem embalagem oscilou do $1^{\circ}$ ao $20^{\circ}$ dia após a colheita, de 57,83 a $34,23^{\circ} ; 58,81$ a $38,21^{\circ} ; 57,65$ a $44,85^{\circ} ; 57,35$

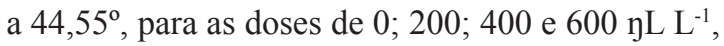
respectivamente. Nos frutos com embalagem, esta oscilação variou de 54,83 a $52,23^{\circ} ; 51,77$ a $49,89^{\circ}$; 52,22 a $50,04^{\circ} ; 51,58$ a $48,86^{\circ}$, nas mesmas doses de 1-MCP testadas (Tabela 1). Independentemente da dose utilizada, os frutos tiveram nítida diminuição da luminosidade ao final do período de armazenamento.

A coloração da casca é uma das características mais importantes na pós-colheita, pois está diretamente relacionada à aceitação do consumidor final. Silva e Muniz (2011), testando a qualidade em frutos de atemoia cv. Gefner, em dois estádios de maturação ("de vez" e maduro), verificaram que o tempo de armazenamento influenciou significativamente sobre todos os atributos de qualidade de atemoia, inclusive na coloração da casca, nos dois estádios de maturação avaliados. $\mathrm{O}$ escurecimento que ocorre durante o período de armazenamento é consequência do amadurecimento dos frutos.

De acordo com Viviani e Leal (2007), a atmosfera modificada pode propiciar redução da respiração dos frutos, diminuindo os níveis de $\mathrm{O}_{2}$ e elevando os níveis de $\mathrm{CO}_{2}$ dentro da embalagem. Dessa forma, o fruto diminui seu processo respiratório, reduz a produção de energia para os demais processos bioquímicos e fisiológicos, retardando, assim, o amadurecimento.

Sugere-se que novos estudos utilizando a atmosfera modificada e sua combinação com outras concentrações de 1-MCP sejam testados por maior período. Dessa forma, a vida útil pós-colheita dos frutos de atemoia 'Gefner' poderia ser ainda maior, reduzindo as perdas durante o armazenamento, e consequentemente, provocando menor risco para os produtores e comerciantes. 
TABELA 1 - Equações de regressão referente às variáveis avaliadas em atemoia cv. Gefner em função de diferentes concentrações de 1-MCP, com e sem embalagem de polietileno de baixa densidade, associadas à refrigeração.

\begin{tabular}{|c|c|c|c|c|}
\hline Variáveis & Embalagem & 1-MCP $\left(\eta L^{-1}\right)$ & Equação (x=época) & $\mathbf{R}^{2}$ \\
\hline \multirow{8}{*}{$\begin{array}{c}\text { Perda de } \\
\text { biomassa fresca }\end{array}$} & \multirow{4}{*}{ Sem } & 0 & $y=0,0273 x^{2}+0,2539 x+0,6798$ & $0,95 * *$ \\
\hline & & 200 & $y=0,0326 x^{2}-0,087 x+0,5476$ & $0,96 * *$ \\
\hline & & 400 & $y=0,0285 x^{2}-0,1067 x+0,3008$ & $0,98 * *$ \\
\hline & & 600 & $y=0,017 x^{2}-0,0048 x+0,2169$ & $0,98 * *$ \\
\hline & \multirow{4}{*}{ Com } & 0 & $y=0,0052 x^{2}+0,2706 x+0,2508$ & $0,98 * *$ \\
\hline & & 200 & $y=0,0071 x^{2}+0,1115 x+0,1606$ & $0,98 * *$ \\
\hline & & 400 & $y=0,0071 x^{2}+0,0408 x+0,204$ & $0,95 * *$ \\
\hline & & 600 & $y=0,0055 x^{2}+0,0217 x+0,1667$ & $0,94 * *$ \\
\hline \multirow{8}{*}{ Firmeza de frutos } & \multirow{4}{*}{ Sem } & 0 & $y=0,574 x^{2}-16,86 x+123,6$ & $0,91 * *$ \\
\hline & & 200 & $y=0,418 x^{2}-14,43 x+135,3$ & $0,98 * *$ \\
\hline & & 400 & $y=0,284 x^{2}-12,37 x+145,5$ & $0,95 * *$ \\
\hline & & 600 & $y=0,276 x^{2}-12,13 x+148,4$ & $0,95 * *$ \\
\hline & \multirow{4}{*}{ Com } & 0 & $y=-0,142 x^{2}-3,084 x+135,0$ & $0,98 * *$ \\
\hline & & 200 & $y=-0,133 x^{2}-2,130 x+136,0$ & $0,98 * *$ \\
\hline & & 400 & $y=-0,212 x^{2}+0,364 x+132,3$ & $0,98 * *$ \\
\hline & & 600 & $y=-0,131 x^{2}-0,952 x+140,6$ & $0,93 * *$ \\
\hline \multirow{8}{*}{ Produção de $\mathrm{CO}_{2}$} & \multirow{4}{*}{ Sem } & 0 & $y=-0,614 x^{2}+12,53 x+40,01$ & $0,57 * *$ \\
\hline & & 200 & $y=-0,506 x^{2}+10,94 x+33,43$ & $0,68 * *$ \\
\hline & & 400 & $y=-0,424 x^{2}+9,833 x+27,02$ & $0,86^{* *}$ \\
\hline & & 600 & $y=-0,360 x^{2}+8,807 x+22,76$ & $0,92 * *$ \\
\hline & \multirow{4}{*}{ Com } & 0 & $y=-0,303 x^{2}+9,959 x+14,05$ & $0,76^{* *}$ \\
\hline & & 200 & $y=-0,179 x^{2}+7,213 x+14,68$ & $0,84 * *$ \\
\hline & & 400 & $y=-0,118 x^{2}+5,854 x+14,57$ & $0,89 * *$ \\
\hline & & 600 & $y=-0,043 x^{2}+4,263 x+13,99$ & $0,94 * *$ \\
\hline \multirow{8}{*}{$\begin{array}{l}\text { Produção de } \\
\text { etileno }\end{array}$} & \multirow{4}{*}{ Sem } & 0 & $y=-0,384 x^{2}+10,65 x-4,014$ & $0,66^{* *}$ \\
\hline & & 200 & $y=-0,367 x^{2}+9,821 x-4,403$ & $0,66^{* *}$ \\
\hline & & 400 & $y=-0,322 x^{2}+8,803 x-4,101$ & $0,69 * *$ \\
\hline & & 600 & $y=-0,336 x^{2}+8,779 x-4,514$ & $0,69 * *$ \\
\hline & \multirow{4}{*}{ Com } & 0 & $y=-0,390 x^{2}+9,284 x+8,204$ & $0,86^{* *}$ \\
\hline & & 200 & $y=-0,287 x^{2}+7,450 x+2,102$ & $0,99 * *$ \\
\hline & & 400 & $y=-0,276 x^{2}+6,885 x+2,816$ & $0,99 * *$ \\
\hline & & 600 & $y=-0,277 x^{2}+6,742 x+1,828$ & $0,98 * *$ \\
\hline \multirow{8}{*}{ Luminosidade } & \multirow{4}{*}{ Sem } & 0 & $y=-1,18 x+57,83$ & $0,96 * *$ \\
\hline & & 200 & $y=-1,03 x+58,81$ & $0,87 * *$ \\
\hline & & 400 & $y=-0,64 x+57,65$ & $0,78 * *$ \\
\hline & & 600 & $y=-0,64 x+57,35$ & $0,59 * *$ \\
\hline & \multirow{4}{*}{ Com } & 0 & $y=-0,13 x+54,83$ & $0,51 * *$ \\
\hline & & 200 & $y=-0,050 x^{2}+0,906 x+51,77$ & $0,96^{* *}$ \\
\hline & & 400 & $y=-0,049 x^{2}+0,871 x+52,22$ & $0,94 * *$ \\
\hline & & 600 & $y=-0,061 x^{2}+1,084 x+51,58$ & $0,87 * *$ \\
\hline \multirow{8}{*}{ Ângulo Hue ${ }^{o}$} & \multirow{4}{*}{ Sem } & 0 & $y=-0,086 x^{2}-0,609 x+101,9$ & $0,96^{* *}$ \\
\hline & & 200 & $y=-0,050 x^{2}-0,726 x+103,5$ & $0,91 * *$ \\
\hline & & 400 & $y=-0,059 x^{2}+0,058 x+101,0$ & $0,99 * *$ \\
\hline & & 600 & $y=-0,087 x^{2}+0,566 x+101,9$ & $0,99 * *$ \\
\hline & \multirow{4}{*}{ Com } & 0 & $y=-0,280 x+102,2$ & $0,65 * *$ \\
\hline & & 200 & $y=-0,261 x+102,4$ & $0,71 * *$ \\
\hline & & 400 & $y=-0,182 x+101,9$ & $0,56^{* *}$ \\
\hline & & 600 & $y=-0,037 x^{2}+0,802 x+97,56$ & $0,96 * *$ \\
\hline
\end{tabular}




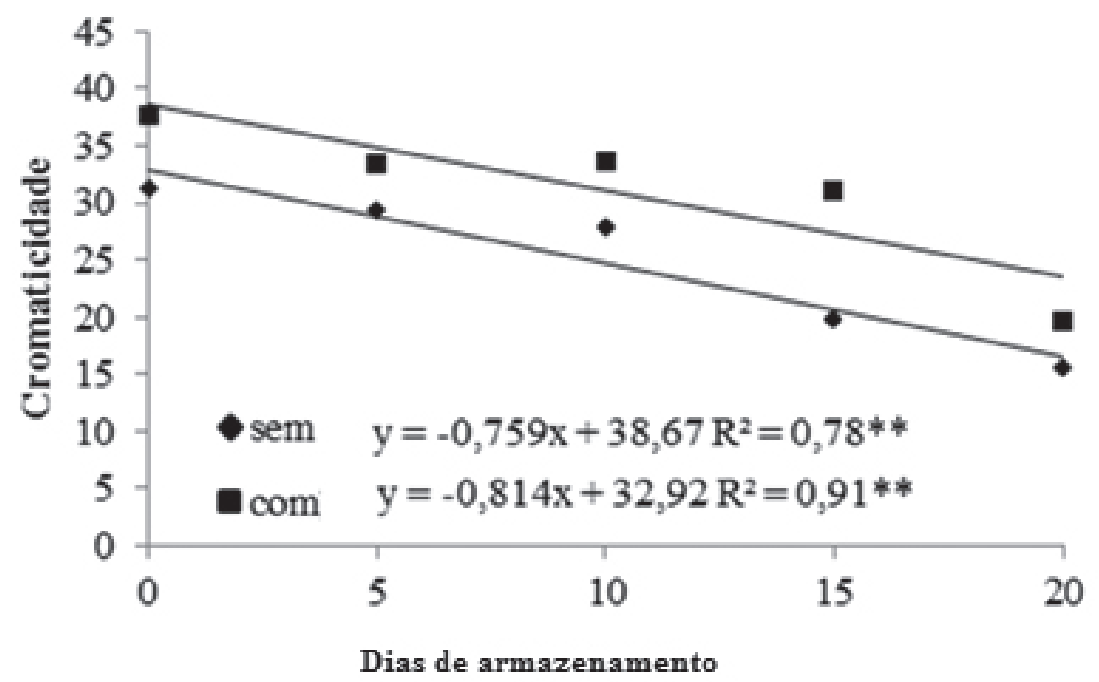

FIGURA 1 - Cromaticidade em atemoias cv. Gefner, sem e com embalagem de polietileno de baixa densidade (PEBD), mantidas a $15 \pm 1{ }^{\circ} \mathrm{C}$ e $94 \% \pm 5,0 \%$ de UR, em função dos dias após a colheita.

\section{CONCLUSÕES}

O uso da atmosfera modificada, associada ou não ao 1-MCP, é eficiente no atraso do amadurecimento de atemoia cv. Gefner, mantendo a conservação de sua qualidade física até o $20^{\circ}$ dia após a colheita. Dentre as doses de 1-MCP utilizadas, a de $600 \eta \mathrm{L} \mathrm{L}^{-1}$ é a que se mostra mais eficaz para as variáveis avaliadas, com exceção do ângulo $H_{u}{ }^{\circ}$, nos frutos com embalagem.

\section{REFERÊNCIAS}

ALVES, E. de O.; STEFFENS, C. A.; AMARANTEI, C. V. T. do; HENDGES, M. V.; ZANARDI, O. Z.; MIQUELOTO, A.; SILVEIRA, J. P. G.; BRACKMANN, A. Amadurecimento de kiwis 'Bruno' submetido ao dano mecânico de impacto e ao tratamento com 1-metilciclopropeno. Bragantia, Campinas, v.69, n. 3, p.753-758, 2010.

BRACKMANN, A.; GASPERIN, A. R. de; BOTH, V.; PAVANELLO, E. P.; SCHORR, M. R. W.; ANESE, R. de O. Armazenamento em atmosfera modificada e controlada de bananas-prata com absorção de etileno. Ciência \& Agrotecnologia, Lavras, v.30, n.5, p.914-919, 2006.

COCK, L. S.; VALENZUELA, L. S. T.; APONTE, A. A. Physical, chemical and sensory changes of refrigerated yellow pitahaya treated preharvest with 1-mcp. Dyna, Medellin, v.80, n.178, p 11-20, 2013.
DANTAS, J. L. L. Catálogo de germoplasma de mamão (Carica papaya L.). Cruz das Almas- BA: Embrapa Mandioca e Fruticultura, 2005. 40 p.

DE MARTINO, G.; VIZOVITIS, K.; BOTONDI, R.; BELLINCONTRO, A.; MENCARELLI, F. 1-MCP control sripening induced by impact injury on a prictos by affecting SOD and POX activities. Postharvest Biology and Technology, Amsterdam, v.39, n.1, p.38-47, 2006.

FIRMINO, A. C.; TOZZE JÚNIOR, H. J.; COSTA, P. N.; FURTADO, E. L. Ceratocystis fimbriata causando murcha em atemoia na região de BotucatuSP. Summa Phytopathology, Botucatu, v.38, n.2, p.171, 2012.

HOJO, E. T. D.; DURIGAN, J. F.; HOJO, R. H. Uso de embalagens plásticas e cobertura de quitosana na conservação pós-colheita de lichias. Revista Brasileira de Fruticultura, Jaboticabal, v.33, n.1, p.377-383, 2011. Numero Especial.

JACOMINO, A. P.; KLUGE, R. A.; BRACKMANN, A.; CASTRO, P. D. C. Controle do amadurecimento e senescência de mamão com 1 metilciclopropeno. Scientia Agrícola, Piracicaba, v.59, n.2, p.303-308, 2002.

LIMA, M. A. C. de; MOSCA, J. L.; TRINDADE, D. C. G. da. Atraso no amadurecimento de atemoia cv. African Pride após tratamento pós-colheita com 1-metilciclopropeno. Ciência e Tecnologia de Alimentos, Campinas, v.30, n.3, p.599-604, 2010. 
MIZOBUTSI, G. P.; Silva, J. M. da; MIZOBUTSI, E. H.; RODRIGUES, M. L. M.; LOPES, R. S.;FERNANDES, M. B.; OLIVEIRA, F. S. Conservação de pinha com uso de atmosfera modificada e refrigeração. Revista Ceres, Viçosa, MG, v.59, n.6, p.751-757, 2012.

MOTA FILHO, V. J. G.; PEREIRA, M. C. T.; NIETSCHE, S.; GUIMARÃES, J. F. R.; MOREIRA, G. B. R.; FERNANDES, T. P. Uso de fitorreguladores no desenvolvimento de frutos na atemoeira (Annona cherimola $\mathrm{x}$ A. squamosa cv. Gefner). Revista Ceres, Viçosa, MG,v.59, n.5, p.636-645, 2012.

SILVA, A. V. C.; ANDRADE, D. G. D.; YAGUIU, P.; CARNELOSSI, M. A. G.; MUNIZ; \& M., \& NARAIN, N. Uso de embalagens e refrigeração na conservação de atemoia. Ciência e Tecnologia de Alimentos, Campinas, v.29, n.2, p.300-304, 2009.

SILVA, A. V. C. da; MUNIZ, E. N. Qualidade de atemoia colhida em dois estádios de maturação. Revista Caatinga, Mossoró, v.24, n.4, p.9-13, 2011.

SOUZA, P. A. D.; FINGER, F. L.; ALVES, R. E.; PUIATTI, M.; CECON, P. R.; MENEZES, J. B. Conservação pós-colheita de melão Charentais tratado com 1-MCP e armazenado sob refrigeração e atmosfera modificada. Horticultura Brasileira, Brasília, v.26, n.4, p.464-470, 2008.

VALERO, D.; GUILLEN, F.; VALVERDE, J. F. 1-MCP use on Prunus spp. to maintain fruit quality and to extend shelf life during storage: a comparative study. 2005. Acta Horticulturae, The Hague, v.2, n.682, p.933-940, 2005.
VIEIRA, M. J.; ARGENTA, L. C.; AMARANTE, C. V. T. do; STEFFENS, C. A.; VIEIRA, A. M. F. D. Preservação da qualidade pós-colheita de kiwi 'Bruno' pelo controle do etileno. Revista Brasileira de Fruticultura, Jaboticabal, v.32, n.2, p.309-406, 2010.

VIEIRA, M. J.;ARGENTA, L. C.; AMARANTE, C. V. T. do; VIEIRA, A. M. F. D.; STEFFENS, C. A. Qualidade pós-colheita de quivi 'Hayward' tratado com 1-mcp e armazenado sob diferentes atmosferas. Revista Brasileira de Fruticultura, Jaboticabal, v.34, n.2, p.400-408, 2012.

VIVIANI, L.; LEAL, P. M. Qualidade pós-colheita de banana-prata-anã armazenada sob diferentes condições. Revista Brasileira de Fruticultura, Jaboticabal, v.29, n.3, p.465-470, 2007.

WATKINS, C. B. The use of 1-methylcyclopropene (1-MCP) on fruits and vegetables. Biotechnology Advances, Waterloo, v.24, n.4, p. 389-409, 2006.

ZONTA, E. P.; MACHADO, A. A.; SILVEIRA JÚNIOR, P. Sistema de análises estatísticas para microcomputadores (SANEST). Pelotas: Universidade Federal de Pelotas, 1984. 151 p. 